\title{
An Authoring Tool for Movies in the Style of Heider and Simmel
}

\author{
Andrew S. Gordon and Melissa Roemmele \\ Institute for Creative Technologies \\ University of Southern California \\ Los Angeles, CA USA \\ \{gordon, roemmele\}@ict.usc.edu
}

\begin{abstract}
Seventy years ago, psychologists Fritz Heider and Marianne Simmel described an influential study of the perception of intention, where a simple movie of animated geometric shapes evoked in their subjects rich narrative interpretations involving their psychology and social relationships. In this paper, we describe the Heider-Simmel Interactive Theater, a web application that allows authors to create their own movies in the style of Heider and Simmel's original film, and associate with them a textual description of their narrative intentions. We describe an evaluation of our authoring tool in a classroom of 10th grade students, and an analysis of the movies and textual narratives that they created. Our results provide strong evidence that the authors of these films, as well as Heider and Simmel by extension, intended to convey narratives that are rich with social, cognitive, and emotional concerns.
\end{abstract}

\section{Introduction}

Heider and Simmel [9] describe a fascinating study that continues to capture our imagination today. In this work, Fritz Heider and Marianne Simmel prepared a short, 90 second animated film depicting the movements of two triangles and a circle around a rectangle with a section that opened and closed as if it was a door. 114 undergraduate subjects were divided into three groups. The first group was shown the film, and then asked simply to "write down what happened in the picture." The second group was instructed to interpret the movements of the figures as actions of persons, and then answer 10 questions concerning the behavior of the triangles and circle. The third group watched the film in reverse, and then asked to answer a subset of the behavioral questions.

Nearly every subject in the first group described the events in anthropomorphic terms, typically involving a fight between two men (the triangles) over a woman (the circle). The narratives of these subjects employ mentalistic phrases: the girl hesitates, she doesn't want to be with the first man, the girl gets worried, is still weak from his efforts to open the door, they finally elude him and get away, he is blinded by range and frustration. The second group of subjects further explained these anthropomorphic interpretations: the larger triangle is an aggressive bully, the smaller triangle is heroic, the circle is helpless, and the 
actions of the smaller triangle and circle lead to their successful escape from the larger triangle, sending him into a rage for being thus thwarted. The third group of subjects, seeing the film in reverse, also interpreted the movements as human actions. Here the variations in narrative were much greater: a man resolved to his fate is disrupted by a woman accompanied by evil incarnate, a prisoner thwarts a murder attempt and then escapes, and a mother chastises a father over the behavior of their child.

What were these subjects thinking? Why did they attribute mentalistic causes to the observable behavior? What cognitive mechanism accounts for these interpretations? Fritz Heider's own explorations of these questions culminated in the writing of his influential book, The Psychology of Interpersonal Relations 8 . Here, Heider motivates the role of commonsense psychology in behavior explanation, where perception of action is integrally tied to a conceptual network of beliefs, desires, sentiments, and personality traits that serve as the invariant dispositional properties that underlie social phenomena. In Heider's view, subjects ascribed mental states to the moving shapes in his film in the same way that they naturally do in everyday human-to-human interactions.

In his autobiography, Heider describes the process of crafting the film with Marianne Simmel by manipulating wooden cutouts on a backlit screen, and his pleasure with the final product:

It took us about six hours, working in this exposure-by-exposure fashion, to make a film that gives a perception of lively movement and takes about three minutes to project. I still remember how pleased I was at my first showing of the film. And it has been impressive the way almost everybody who has watched it has perceived the picture in terms of human action and human feelings. [7]

Heider's writings provide us with no information about the narrative that he intended to convey through this animation, or even that such an intention was present. Still, it must be remembered that the conventions of animated storytelling [15] were already well established in the popular films of the 1940s, which saw the release of Disney's Pinocchio (1940), Fantasia (1940), Dumbo (1941), and Bambi (1942) in the years before Heider and Simmel crafted their anachronistic film. For Heider's subjects, the question of "what happened in the picture" would have been naturally understood as "what story have the animators intended?" From this perspective, however, the experiment of Heider and Simmel leaves many questions unanswered. What sorts of narratives can be successfully conveyed given the limits of the medium? What are the factors that govern the degree of variance in interpretations? How much can successful storytelling be attributed to the the talents of Heider and Simmel, specifically, as animators? Answering these questions requires that we enlist the talents of a larger pool of animators, whose explicit authorial intentions can be compared to audience interpretations.

This paper describes a software solution for creating and narrating movies in the style of Heider and Simmel's film. The Heider-Simmel Interactive Theater 
allows authors to record their own movies by dragging shapes around a two dimensional plane using a mouse or multitouch interface, and then associate with them a written description of their narrative intentions. Built as a web application, users' movies are viewed by other users, who can share their own written interpretations. After presenting the software and its capabilities, we describe an evaluation of this authoring tool by students in a 10th grade public school classroom in Los Angeles, using school-issued tablet computers as a hardware platform. We then provide an analysis of these students' animations and written narratives, to better understand the conceptual scope of the narratives that these films were meant to convey.

\section{The Heider-Simmel Interactive Theater}

The Heider-Simmel Interactive Theater 11 is a web-based authoring tool designed to allow users to author their own movies in the style of Heider and Simmel's film, and to author textual narratives of their own films and those of other users. Unlike professional animation or movie-editing software that strive for realism and high production quality, the design goal for this application was to enable novice users to quickly make short (90 second) movies without the need for instruction of any sort. We achieved this goal through a combination of intuitive user interface design and by limiting the movies' characters and props to exactly those seen in Heider and Simmel's original film: one large triangle, one smaller triangle, one circle, and one box with a movable door. Each of these elements appear in every movie, which can be fully encoded as a time-indexed array of position and rotation parameters for the three characters and the door. Figure 1 shows a user creating a movie using this software on a tablet computer.

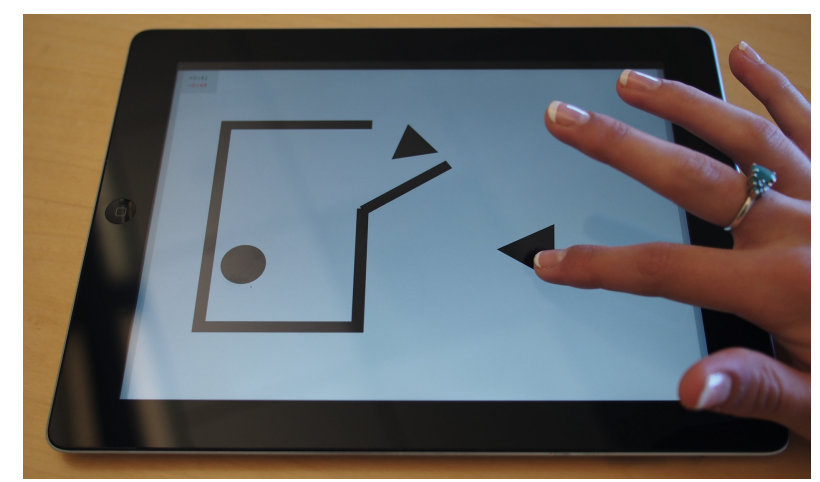

Fig. 1. The Heider-Simmel Interactive Theater

Architecturally, this web application consists of client-side hypertext markup and scripts, making heavy use of Scalable Vector Graphics, and a server-side

\footnotetext{
${ }^{1}$ Accessible online at http://hsit.ict.usc.edu
} 
application interface and database to store user login accounts and movie data. The client-side application allows users to manipulate characters using either a mouse or touch interface, enabled by handling web browser-based touch events. This architectural design simplifies the deployment of the application; users access the application using the web browser on their desktop or tablet computers. As all movies and textual narratives are stored centrally on our servers, this provides us with an ever-increasing amount of data to be analyzed in our research on visual storytelling.

\subsection{Object Manipulation}

The main authoring screen of this application is realized as a full-screen Scalable Vector Graphics (SVG) object in the Document Object Model of a web page. The white stage and black shapes from Heider and Simmel's original film are represented as elements of this SVG object, with object properties that specify their rotation and translation transformations. Compared to Heider and Simmel's original, these elements are scaled slightly larger, making it easier for a user to place their finger directly on top of an object when using a tablet computer, given contemporary screen sizes. Even with this concession, we found it to be ergonomically difficult for a user to place two fingers on a single shape, which is required when using a common interface convention for simultaneous rotation and translation of two dimensional objects. Accordingly, it was necessary to develop a capability for expressively dragging a character across the stage using only a single finger, or via a traditional mouse interface.

Our custom 1-pointer solution for simultaneous rotation and translation consists of two parts, the "sticky finger" and the "slippery center." Dragging is initiated on mouse-down or touch-start browser event handlers attached to each draggable SVG element. As the user subsequently drags their mouse or finger, the position of the SVG object is updated such that the pointer's location on the object is the same as when the drag was initiated (the "sticky finger"), and the new center point of the object lies on the line segment between its previous location and the new location of the pointer, as in Figure 2(a). By updating the SVG position at the rate of incoming pointer events, this approach yields fluid and intuitive manipulation of each draggable object in most cases. However, if the dragging is initiated near the center point of the object, small and uncontrollable movements of the pointer will tend to induce large, sporadic rotations. We found a simple solution to this problem, which is to suppress the initiation of dragging until the pointer has been dragged sufficiently far away from the center point of an object (the "slippery center"), as in Figure 2(b). The latency in an object's movement is largely imperceptible, and the effect is that every attempt to drag an object affords a degree of expressive control.

We crafted a specialized dragging routine for the door of the box to allow it to be rotated nearly 180 degrees in either the open or closed direction. We made no efforts to detect or react to object collisions, or to model inertia or other physical properties of moving masses. Unfortunately, these choices prohibit users 


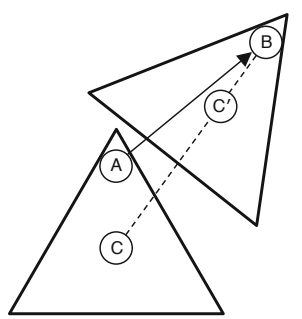

(a)

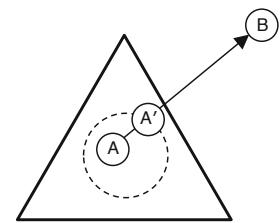

(b)

Fig. 2. Dragging gestures $\overrightarrow{A B}$, showing (a) positioning of new center point $C^{\prime}$ on $\overline{C B}$, and (b) suppression of dragging from center region, yielding drag gesture $\overrightarrow{A^{\prime} B}$

from recreating the climactic ending of Hieder and Simmel's original film, where the larger triangle busts through the walls of the box from the inside. We intend to support this option in future versions of the authoring tool.

\section{$2.2 \quad$ Recording and Editing Movies}

Much of the complexity of conventional video production software relates to the recording and editing process. For the Heider-Simmel Interactive Theater, our design goal was to provide users with some editing abilities, but to do so with an intuitive user interface that required no instruction.

When creating movies, users are first see the stage with characters in fixed starting positions. Recording begins as soon as a user starts to drag a character, using their mouse pointer or a finger. From this moment, a timer display in the corner of the screen becomes active, displaying both the elapsed time and available time remaining for the performance, limited to 90 seconds. In the simplest case, a user simply moves the various characters to record their movie until 90 seconds have elapsed.

If the user clicks on the timer display, or when the timer reaches 90 seconds, a movie editing toolbar is displayed (Figure 3). Similar to conventional playback controls for digital video, this editing toolbar has a slider that moves from left to right to indicate the current time point in the movie. To the left of this slider is a (green) recording trail, indicating the portion of the duration that contains recorded data. Dragging the slider left or right along this toolbar allows the user to scrub through their recording, causing the characters to move back to their positions as recorded at each time point. Clicking the slider starts playback from the selected time point.

Fig. 3. Movie editing toolbar 
If the user initiates dragging during playback, the recording begins again from that moment forward. This action erases all subsequent recorded data, which is reflected in the recording trail. This interface behavior affords a simple form of incremental, linear editing of a recorded performance. To make a change, the user moves the slider to the time point in their recording that they would like to edit, and then creates a revised movie from that point onward. Although limited, this approach eliminates the complex interface controls necessary in nonlinear video editing, e.g. to support splices and joins.

Two additional controls, represented as (white) polygons on either end of the editing toolbar, allow users to trim off durations from the beginning and ending of their recording. These "trim blocks" can be dragged left and right, up until the current position of the slider, to specify new start and end times for a recorded performance. The start time trim block is particularly useful for creating movies where the characters begin in novel positions. To do so, the user takes a few seconds at the beginning of their recording to position their characters as desired, then trims this duration off during editing.

\subsection{Narrating Movies}

The Heider-Simmel Interactive Theater allows users to associate their own textual narration with their movies, and to offer textual interpretations of the movies of other users. When watching another user's movie, we first ask the viewer to give the film a "star rating" from one to five, and then describe their interpretation of the events in the movie. When finished, the viewer is shown the author's own textual narration, for comparison. Each viewer's interpretation also becomes visible to the author to review on subsequent visits to this web application.

\section{Evaluation}

To assess the usability of our authoring tool design, we conducted a user evaluation with 10th grade students from Alliance Health Services Academy, a charter high school providing public education to students in a South Los Angeles neighborhood. We conducted this evaluation with 23 students during a twohour classroom period intended specifically to prepare students for the California High School Exit Exam. Our exercise with this classroom was scheduled at the end of the academic year, after state-mandated achievement tests, so as not to conflict with their normal educational curriculum. The majority of these 23 students were 15 or 16 years old, and included all of the 10th grade students in the school receiving special education as part of an Individualized Education Program (IEP). Each student in this classroom uses an Apple iPad through an initiative of the Los Angeles Unified School District that began in 2013 aimed at providing tablet computers to every student. These iPads are distributed to students each morning, and later returned to school administrators to be recharged overnight. 
Three members of our research team conducted this evaluation, with the assistance of this classroom's teacher. On this teacher's advice, we offered no background information about Heider and Simmel's original film, and nearly no instruction on how to use our software. After brief introductions to our team, we handed out index cards with pre-established usernames and passwords for each student, along with the URL of the Heider-Simmel Interactive Theater to enter into their iPad's web browser. Students were then asked to perform three tasks, and given roughly 15 minutes for each. First, we asked to create their own creative movies, depicting any storyline of their choosing, using the authoring interface described in the previous section. Students completed this task easily, and many used the allotted time to create additional movies. During this time, our research team answered students' questions and monitored the classroom for problems, which were minimal. Second, we asked students to write a textual narrative that described the events depicted in their movies, using the touchscreen keyboard that appears on the iPad's browser screen. Third, we asked students to write their interpretations of movies created by other users of our application, selected randomly from all previous recordings. Of the three tasks, this one was the least successful, as students were largely only interested in seeing the films created by their fellow classmates. As a consequence, they spent an inordinate amount of time clicking through random movies until they found one associated with a username assigned to their peers.

After these tasks were completed, the teacher of this classroom directed a classroom exercise for the remainder of the period. Here, students were selected to present their movies to the whole class, by connecting their iPads to a classroom video projector, and tell the class the narrative they intended. Our research team was struck by the high degree of creativity exhibited by this group. To better understand the ways that our tool was used by these students, we subsequently conducted analyses of both the recorded movie data and the students' textual narratives, described below.

\subsection{Analysis of Movie Recordings}

We analyzed the usage of two user interface features intended to aid the authoring and editing of movies. First, we analyzed usage of the multitouch interface, which enabled authors to simultaneously animate two or more shapes. We examined each of the 43 movies authored by students in our evaluation, specifically looking for evidence of multitouch animations. Our encoding of the movie data made it simple to detect when shapes were moving simultaneously. By iterating through each time point in the recording, we detected multitouch activity when more than one shape changes position between consecutive time points. We found that 16 of 23 students $(70 \%)$ used the multi-touch authoring capability, and these students authored 23 multi-touch movies. Since students largely authored animations independently, we interpret this result as evidence of an intuitive design for character manipulation.

Second, we analyzed the usage of functionality in the movie edit bar, shown in Figure 3. We had provided students almost no instruction on the use of the 
edit bar, so we were particularly interested in seeing evidence of successful movie editing. However, our incremental linear editing approach, where edits are made by recording over trailing portions of the user's movie, leaves no evidence of edits in the movie data. However, we could detect the use of the "trim blocks" that enable authors to delete portions from the beginning and ending of movies, shown as white polygons in Figure 3. Use of the beginning trim block can accurately be detected by examining the position of characters in the first frame of users' movies. Cases where characters are not in their default positions indicates that the author had used the trim block to adjust the start frame of the movie. We found that 4 of 23 authors (17\%) did this type of editing, and they contributed 5 movies with an edited start time. We interpret this result as weak evidence for an intuitive design for movie editing, particularly given that this feature was not explicitly communicated to students.

\subsection{Analysis of Textual Narratives}

Thirty-four of the 43 movies created by students in our experiment were narrated by their authors, who typed these short, paragraph-sized texts directly into our user interface using their iPads. A wide range of topics and descriptive elements appeared in these narratives, as seen in the following three examples:

The triangles are the brother and the circle is the sister. The sister was annoyed by her brothers so she went to her room since she didn't want to be bothered. So her brothers went after her bugging her even more. Once they got out of her room, she went to go apologize.

The three musketeers must fight the evil box. While Mr. Circle goes to fight the evil box, the evil box eats Mr. Circle. Mr. Small triangle then tries to help his friend, but gets eaten in the processes as well. It's all up to Mr. Big triangle to save the day. Mr. Big triangle fights the evil box. He then kills the evil box and saves both his friends, and they lived happily ever after.

A girlfriend is upset at her boyfriend for ignoring her. He is always talking to his bestfriend and pays her no mind until it's too late. Once he notices her she already has a really big attitude, so he gives up on trying to make up with her. But she forgives him and they make up anyway.

To better understand the diversity of the narrative elements used by these students, we conducted a series of lexical analyses to identify patterns in the types of characters, setting, and actions that were described.

First, we analyzed references to characters in these narratives. The HeiderSimmel Interactive Theater has four objects that can be animated: three shapes (circle, little triangle, and big triangle), and the door of the box. We began by identifying and counting distinct characters mentioned in each narrative, finding either four (3 narratives), three (20 narratives), two (10 narratives), or one character (1 narrative) referenced in the text. When four characters were 
mentioned, the authors either referred to characters not explicitly shown in the animation or treated the box as a character (the evil box, in the second example above). Of the 93 characters referenced in these narratives, most (58) were referred to by their respective shape names (e.g. "circle" or "triangle"), and the two triangles were distinguished by their relative size, i.e. calling one the "big" or "large" triangle and the other the "small" or "little" triangle. We discerned gender assignments for 35 characters, either by personal pronouns or gender-specific roles (e.g. "boyfriend", "sister"), and found male characters more frequent (27) than female characters (8). The most commonly expressed relationship between characters was friendship, as indicated by the keywords "friend" or "best friend", which occurred in 13 narratives. Three narratives described a dating relationship ("boyfriend", "girlfriend"), 3 narratives described a sibling relationship ("brother", "sister"), and 2 narratives described a "teacher" and "student" relationship. Interestingly, the author appeared as a character in 4 narratives, identified through the use of first-person personal pronouns.

Second, we analyzed references to the locations and props in these narratives. The majority of narratives (20) explicitly mentioned locations or props, some of which refer to on-stage objects. We were particularly interested in how authors referred to the box on the stage, given its ambiguity. Authors made frequent use of terms indicating a walled structure with a door, including "house," "home," "door," "room," "building," "entrance," and "exit." Two narratives interpreted the animated circle as a "ball" rather than a character. Further creativity is evidenced by a wide variety of other locations and props included in these narratives, including "roof," "pool," "safe," "hallway," "money," "corner," "lock," and "cave." These locations and props evoke a variety of intended activity contexts and situations, which are only sometimes explicated in the text. For example, one author writes: "First, their is two triangles and a circle going over to a house which a friend is throwing a party. "

Third, we analyzed references to actions performed by characters in these narratives. The subjects in Heider and Simmel's 9 original experiment reportedly viewed the shapes as human characters that physically manipulated their environment, engaged in social interaction with other characters, and experienced psychological states involving thought and emotion. In analyzing the range of actions and experiences in our students' narratives, we aim to characterize the degree to which these elements also exist in the narratives intended by the authors of these animations, and by Heider and Simmel as well by extension. For this analysis, we catalogued each narrative according to its predicates, i.e. phrases indicating an action or experience on the part of the character typically verbs (e.g. "chase", "talk") or adjectives ("happy", "in love"). With each narrative containing a mean of 52 words, there were 236 total predicates across all narratives, or 6.9 predicates per narrative on average. We classified these predicates into five different high-level categories. First, we defined "physical predicates" as actions involving physical interaction between a single character and its environment (e.g. "walk", "enter"). Second, "physical-social predicates" are actions involving physical interaction between at least two characters (e.g. "fight", "fol- 
low"). Third, "psychological-social predicates" are also actions involving social interaction between at least two characters, but these actions rely strongly on human-like psychological capacities (e.g. "talk", "convince"). Fourth, "perceptual predicates" involve a character experiencing human-like sensory abilities and cognition (e.g. "see", "think"). Finally, "emotional predicates" involve a character experiencing human-like emotions, desires, and intentions by a character ("want", "get angry"). In general, these predicate categories are listed in the order of increasing psychological attribution on the part of the author. The former categories reflect behavior more directly observed from the movements in the animations. In contrast, the latter categories reflect experiences not easily observed in the animations, but elaborated by the author based on insight into the characters' psychology.

Table 1. Predicates used in textual narratives

\begin{tabular}{|c|c|c|c|}
\hline category & predicates & count & narratives \\
\hline physical & $\begin{array}{l}\text { come/go, jump, trapped, open/close, } \\
\text { lock/unlock, wait, take, turn, transform, } \\
\text { relax, throw, fall, hit, escape, shake, } \\
\text { sit/stand, knock }\end{array}$ & $75(32 \%)$ & $21(62 \%)$ \\
\hline physical-social & $\begin{array}{l}\text { fight, capture/restrain, join/approach, } \\
\text { evade, rescue, play, kill, chase, hide, hit, } \\
\text { help, block, lead/follow, interfere }\end{array}$ & $47(20 \%)$ & $22(65 \%)$ \\
\hline psycho-social & $\begin{array}{l}\text { talk, socialize, bother, befriend/de-friend, } \\
\text { convince, intimidate, agree/refuse, invite, } \\
\text { mock, argue, deceive, scream, cry, defend, } \\
\text { laugh }\end{array}$ & $45(19 \%)$ & $18(53 \%)$ \\
\hline perceptual & see, think, ignore, hear, lost, count & $31(13 \%)$ & $15(44 \%)$ \\
\hline emotional & $\begin{array}{l}\text { angry, desire, anxious, sad, annoyed, } \\
\text { happy, tired/alert, love, dislike, serious, re- } \\
\text { act, confused, relieved, give up, injured }\end{array}$ & $38(16 \%)$ & $22(65 \%)$ \\
\hline
\end{tabular}

Table 1 presents statistics for each high-level category, and lists each of the predicates apparent in the text, ordered by frequency from high to low. Of the 236 predicates contained in these narratives, the most frequent predicate, by far, was the come/go predicate in the physical category, which occurred 43 times across 18 narratives. The second most frequent predicate overall was the see predicate in the perceptual category, evident 18 times in 11 narratives. Also frequent was the talk predicate in the psychological-social category, evident 13 times in 8 narratives. Collectively, the diversity of predicates used across these narratives provides strong evidence that authors of movies in the style of Heider and Simmel intend rich narratives that pass well beyond the realm of observable physical actions, and into a wide range of social, psychological, and emotional concerns. 


\section{Discussion}

Our evaluation in a classroom of high-school students provided us with convincing evidence that we had achieved our design objective: The Heider-Simmel Interactive Theater allows novice users to easily author movies intended to convey rich narratives that involve various physical, social, and psychological concerns. By broadening the pool of authors, we can now begin to explore many of the research questions that follow from Heider and Simmel's [9] thought-provoking experiment. What sorts of narratives can be successfully conveyed given the limits of the medium? What are the factors that govern the degree of variance in interpretations? How much can successful storytelling be attributed to the the talents of Heider and Simmel, specifically, as animators? We hope that the evergrowing number of movies and narratives collected through this application will support these analyses in the future. Additionally, it provides a new tool that researchers can use to craft visual stimuli for controlled experiments, particularly for those perception psychologists who use abstract shapes in their studies [15]6]16.

Similar experimental stimuli has also been popular among developmental psychologists, both for studying developmental changes in the perception of causality 4 4/11/14 and in understanding the developmental impairments of autism 210]13. This tool may be useful in psychiatric clinical settings, particularly for children who are predisposed to interact with technology. The Heider-Simmel Interactive Theater may compliment other projective personality measures used in psychoanalysis, such as Rorschach inkblot tests or the Thematic Apperception Test, by allowing patients a means of interacting with ambiguous and unstructured stimuli.

Our experience with 10th grade students also encourages us to explore the educational potential of our application. We expect that the Heider-Simmel Interactive Theater would be useful in language arts curriculum, particularly as a tool for developing creative writing and storytelling skills. In school systems that are embracing tablet computers as educational tools, our application could potentially be used in lesson plans that include a presentation of Heider and Simmel's original research, and the influence of Heider's work on contemporary social psychology.

Our own research interests are toward the use of this application as a datacollection platform for research in artificial intelligence. As in previous research 3]12, we aim to develop algorithms that can automatically interpret and narrate observed behavior, in much the same fashion as the subjects in Heider and Simmel's original study. This authoring tool allows us to collect hundreds or thousands of movie and narration pairs from volunteers, which can be used both to evaluate the performance of our algorithms and to model the relationships between observed action, intentions, and the language used to narrate interpretations.

Last, but not least, our application affords a new form of creative expression for web users of all sorts, enabling interactive digital storytelling through the playful manipulation of geometric shapes. 
Acknowledgments. We thank Ms. Maribel Gonzalez and her 10th grade students at Alliance Health Services Academy in Los Angeles for their participation in this research. This research was supported by ONR grant N00014-13-1-0286.

\section{References}

1. Barrett, H., Todd, P., Miller, G., Blythe, P.: Accurate judgments of intention from motion cues alone: A cross-cultural study. Evolution and Human Behavior 26, 313-331 (2005)

2. Bowler, D., Thommen, E.: Attribution of mechanical and social causality to animated displays by children with autism. Autism 4(2), 147-171

3. Crick, C., Scassellati, B.: Inferring Narrative and Intention from Playground Games. In: Proceedings of the 7th IEEE International Conference on Development and Learning (ICDL 2008), Monterrey, California (August 2008)

4. Dasser, V., Ulbaek, I., Premack, D.: The perception of intention. Science 253, 365-367 (1989)

5. Gao, T., McCarthy, G., Scholl, B.: The Wolfpack Effect: perception of animacy irresistibly influences interactive behavior. Psychological Science 21(12), 1845-1853 (2010)

6. Gao, T., Newman, G., Scholl, B.: The psychophysics of chasing. A case study in the perception of animacy. Cognitive Psychology 59, 154-179 (2009)

7. Heider, F.: The life of a psychologist: An autobiography. Lawrence, KS: University of Kansas Press

8. Heider, F.: The psychology of interpersonal relations. Lawrence Erlbaum Associates, Hillsdale (1958)

9. Heider, F., Simmel, M.: An experimental study of apparent behavior. American Journal of Psychology 13 (1944)

10. Klin, A.: Attributing social meaning to ambiguous visual stimuli in higherfunctioning autism and asperger syndrome: The social attribution task. Journal of Child Psychology and Psychiatry 41(7), 831-846

11. Leslie, A., Keeble, S.: Do six-month-old infants perceive causality? Cognition 25, 265-288 (1987)

12. Pautler, D., Koenig, B., Quek, B.K., Ortony, A.: Using modified incremental chart parsing to ascribe intentions to animated geometric figures. Behavior Research Methods 43(3), 643-665 (2011)

13. Salter, G., Seigal, A., Claxton, M., Lawrence, K., Skuse, D.: Can autistic children read the mind of an animated triangle? Autism 12(4), 349-371

14. Schlottmann, A., Surian, L.: Do 9-month-olds perceive causation-at-a-distance? Perception 28, 1105-1113 (1999)

15. Thomas, F., Johnston, O.: The Illusion of Life: Disney Animation. Hyperion, New York (1995)

16. Tremoulet, P., Feldman, J.: Perception of animacy from the motion of a single object. Perception 29, 943-951 (2000) 